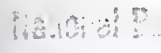

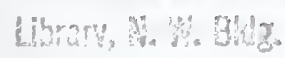

NOV 161949

U. S. DEPARTMENT OF COMMERCE

NATIONAL BUREAU OF STANDARDS

CENTRAL RADIO PROPAGATION LABORATORY

WASHINGTON, D. C.

COMPARISON OF PREDICTIONS OF ATMOSPHERIC

RADIO NOISE WITH OBSERVED NOISE LEVELS

BY E. L. SHULTZ 



\title{
COMPARISON OF PREDIC TIONS OF ATMOSPHERIC RADIO NOISE WITH OBSERVED NOISE LEVELS
}

\author{
E. L. Shultz
}

Abstract-- Two years' data from 16 atmospheric radio-noise measurement stations show sufficient consistencies in the relationship of the noise level and the geographic location of the observing station to indicate that a linear latitude trend approaches a definition of that relationship. The data on 2.5 and 5 Mc during night hours show a fair agreement with the United States Army Signal Corps predictions (RPU-140). An even closer agreement exists between the observed data and a linear latitude relationship for each frequency. The average noise level for the northern hemisphere exhibits a variation, seasonal sine wave with an amplitude of about three units, superposed on a general decreasing trend for the past two years at the rate of about four units per year.

In 1943 the Wave Propagation Committee of the Combined Communications Board, Combined Chiefs of Staff, started investigation of the problems of measurement of atmospheric radio noise. The equipment developed by H. A. Thomas of the National Physical Laboratory, Teddington, England, was approved in August 1944 and went into operation at a few stations by March 1945. By July of that year eight stations were taking measurements. A total of 17 stations, distributed in latitude from $51^{\circ} \mathrm{N}$ to $41^{\circ} \mathrm{S}$, were in operation for all or part of the first two years of the active program (see Fig. 1).

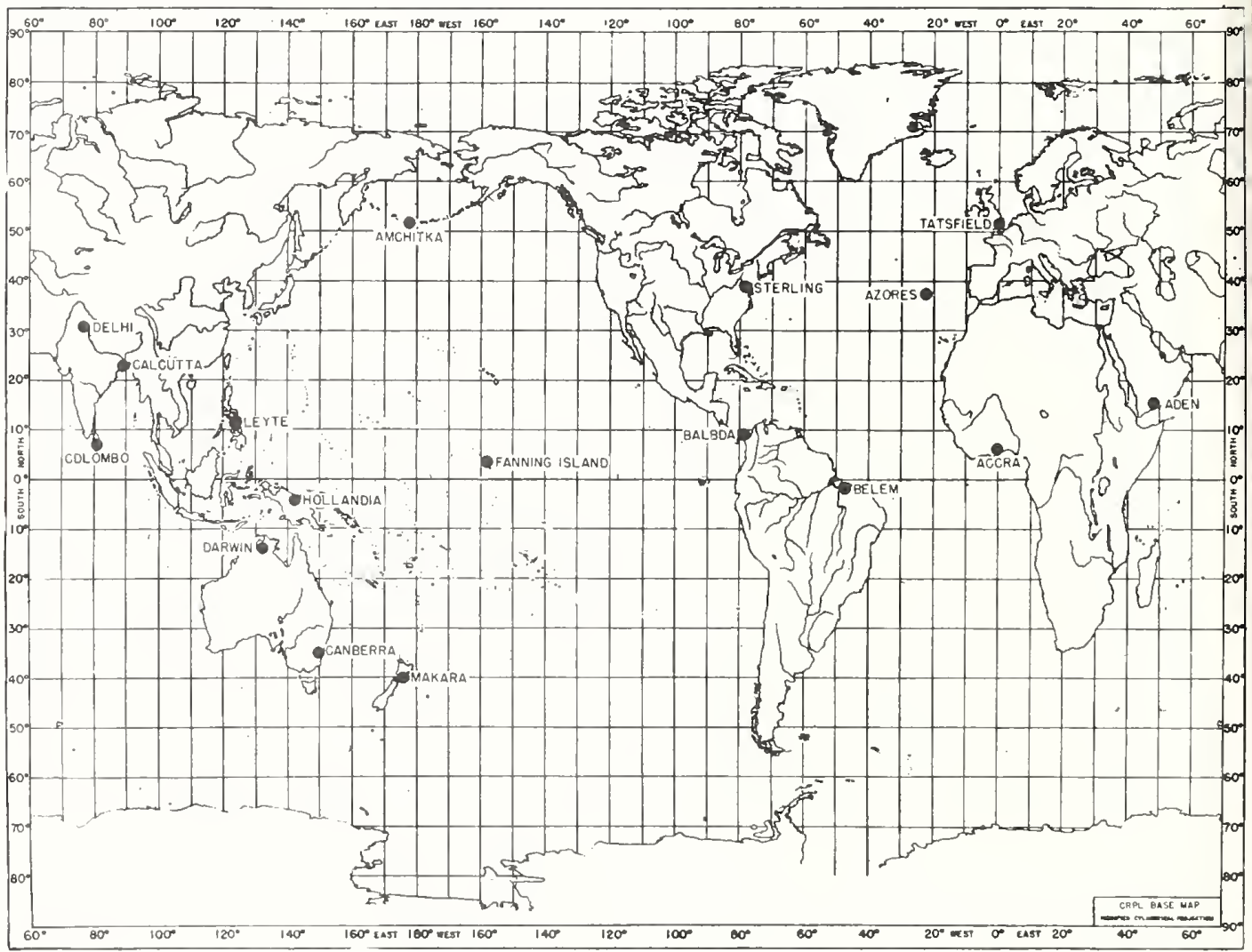

Fig. 1--Locations of stations for measurement of atmospheric radio noise 
The method of measurement of atmospheric noise which has been used in the project attempts to determine directly the minimum field intensity required for a given degree of intelligibility in the presence of atmospheric noise. The observations give the field intensity in log microvolts per meter times 20, hereinafter to be called units, necessary for 95 per cent intelligibility of a tenword-a-minute $\mathrm{cw}$ signal with a ten-kc bandwidth receiver. It requires that the $\mathrm{cw}$ signal from a seyed standard voltage generator be varied in intensity by means of the voltage generator's attenuator until the operator is able to transcribe just 95 per cent of the five-letter groups in the presence of the noise picked up by a 20-foot vertical antenna. Hourly readings of this minimum required field intensity are taken on frequencies as close to $2.5,5,10,15$, and $20 \mathrm{Mc}$ as it is possible to find a clear channel. Set noise level of the equipment imposes a limitation on the natural noise which can be measured. For this reason low measured values of noise, may not represent natural noise but only the lower limit of sensitivity of the equipment. In interpreting the results care was exercised to avold errors arising from this cause.

The noise data are collected and redistributed by three cehtralizing agencies: National Physical Laboratory (NPL), Teddington, England; Australian Radio Propagation Committee (ARPC), Sydney, Australia; and Central Radto Propagation Laboratory (CRPL), National Bureau of Standards, Washington, D. C. Research goes forward at the three laboratories, with CRPL conducting investigations of three aspects of the phenomena... diurnal variation, seasonal variation, and latitude variation.

The most obvious and elementary finding common to the three aspects is that great differences from station to station exist. However, certain systematic characteristics have been revealed, and these characteristics will be discussed in this report.

One of the most useful forms into which the data of the first two years of the project have been cast are shown in Figure 2, which represents daily observations at a fixed hour for one month. The

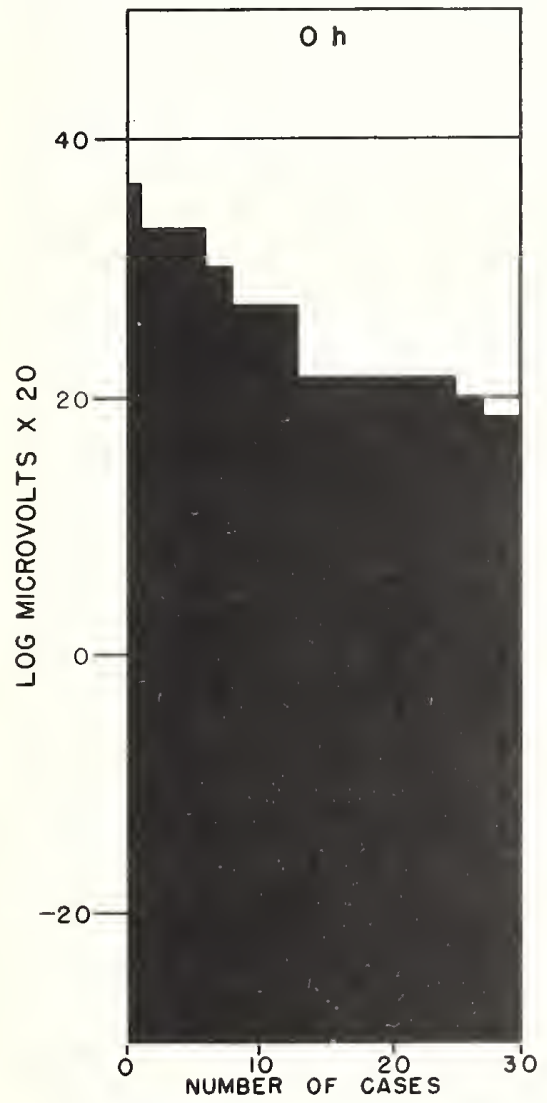

Fig. 2--Polygon showing frequency of occurrence of atmospheric radio noise at various intensities at midnight, Accra, British West Africa, October 1945 ordinates are the required field intensities in $\log$ microvolts per meter times 20 , and the abscissas are the numbers of times each intensity occurs during the month on a descending scale. Figure 2 indicates that at Accra at midnight, during October 1945, a level of 37 units was reached but not exceeded once; 33 , five times; 30 , twice; 27 , five times; 23 , twelve times; 20 twice, and 17 three times. Readily obtainable from this kind of graph are the month's range for this single hour and the median or other percentile values. The third value from the left, one-tenth of the horizontal scale, with an ordinate of 33 units, for example, is the minimum at or below which 90 per cent of all occurrences fall. This is the upper decile value. This 90 per cent time, 95 per cent intelligibility value is functionally the most useful with which we are at present concerned. In this study, because of uncertainties in lower values previously mentioned, only the values of noise which were reached or exceeded 90 per cent of the time were considered, and averages referred to are averages of those values.

In Figure 3, beginning with the hour graphed in Figure 2, 24 successive hourly polygons present a picture of what happened throughout the 24 hours for a given month. This kind of plot shows at a glance the shape of the normal diurnal curve, the magnitude of the diurnal variation, and on what frequencies and at what time of day local storms, characterized by peaks such as those in Figure 3 on $5 \mathrm{Mc}$ at $01^{\mathrm{h}}$ and $02^{\mathrm{h}}$ and on all frequencles except ten Mc at $13^{\mathrm{h}}$ and $14 \mathrm{~h}$, affected noise levels. From enough of these plots some generalizations can be drawn concerning noise at various stations. 


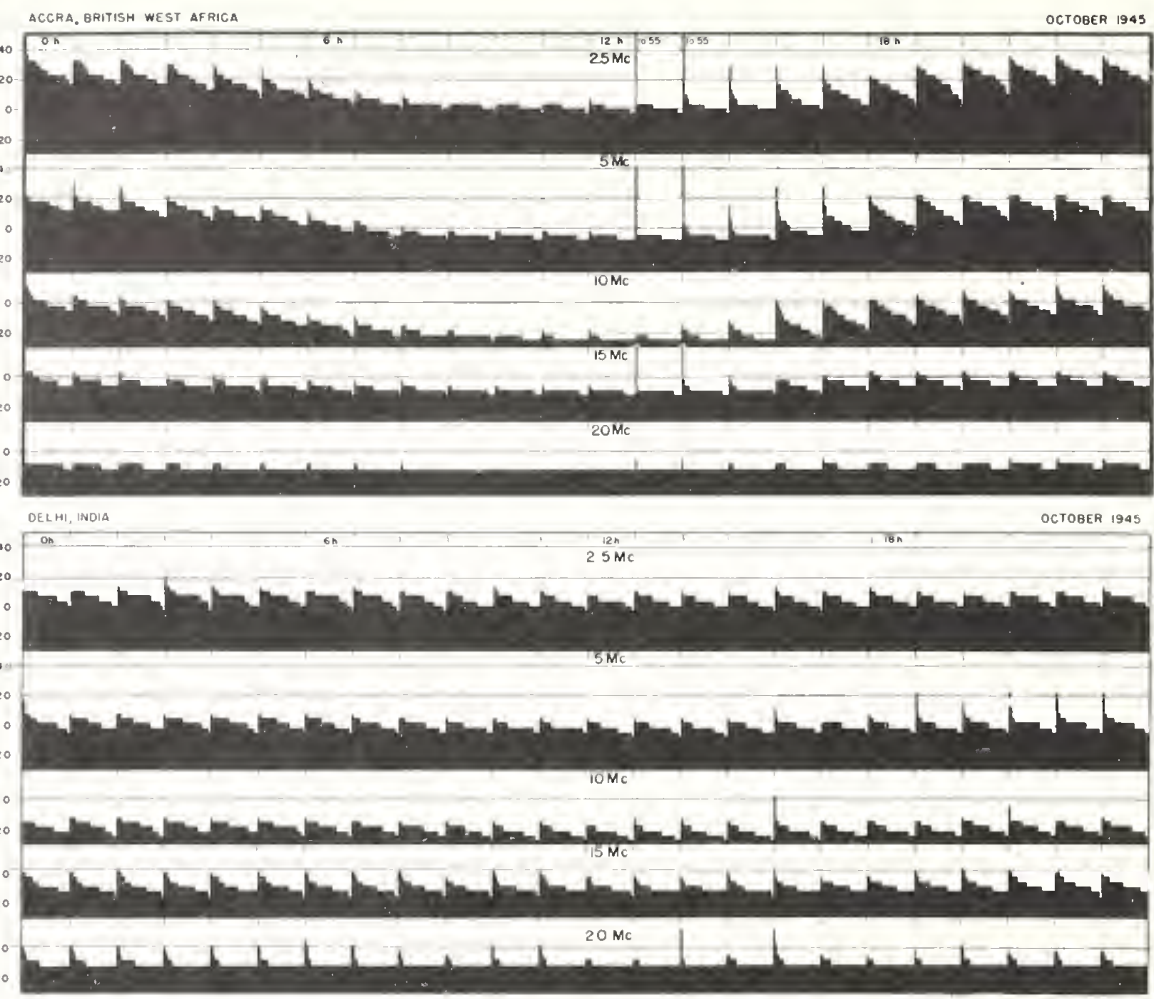

Fig. 3--Polygons showing frequency of occurrence of atmospheric radio noise at various intensities at midnight, Accra, British West Africa, and Delhi, India, October 1945

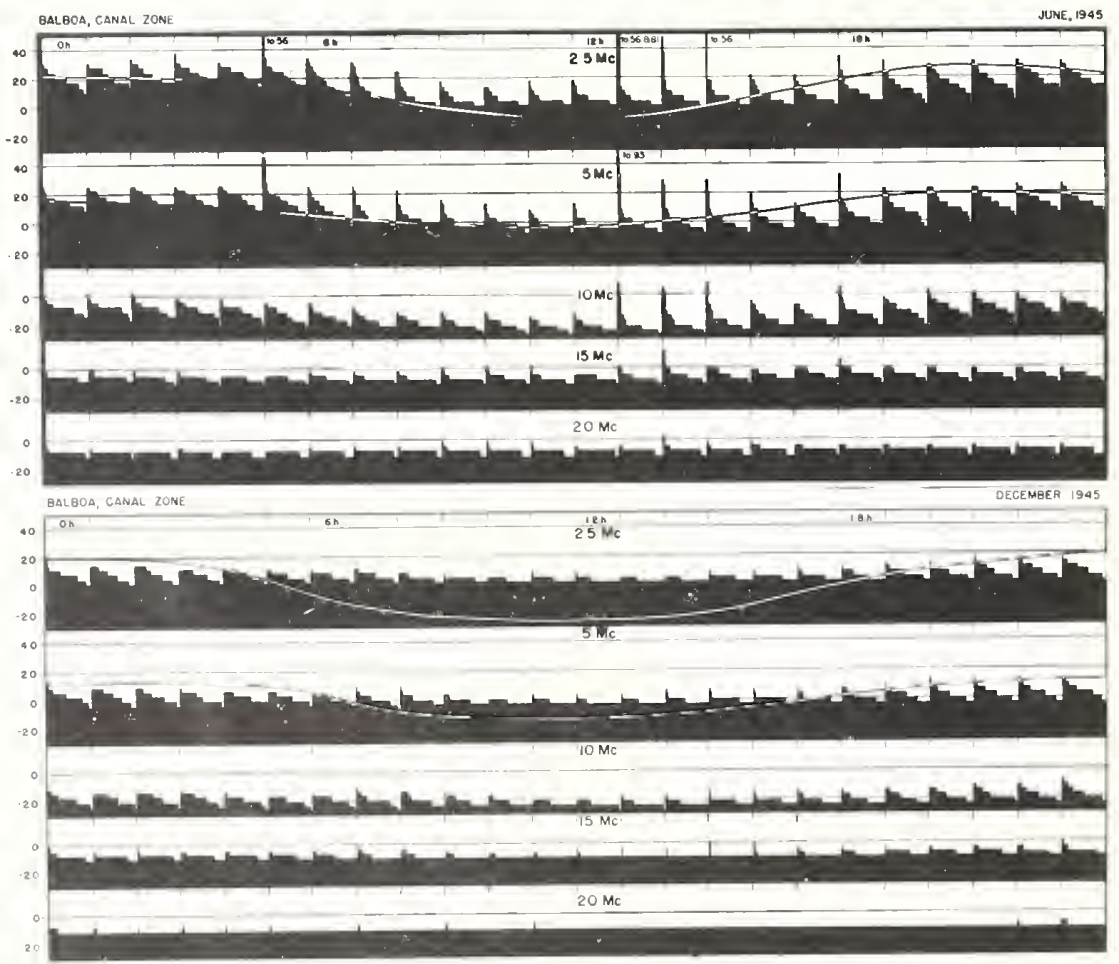

Fig. 4--Polygons showing frequency of occurrence of atmospheric radio noise at various intensities for each hour at Balboa, Canal Zone, June and December 1945 
Data from two stations, represented in Figure 3, were selected to give an idea of the nature of durnal variation in atmospheric radio noise. They show that on $2.5 \mathrm{Mc}$ a drop of about 30 units occurred at Accra from midnight to noon. The times of the most sudden change in level at Accra, about sunrise, which is just before $06^{\mathrm{h}}$ in October, and near sunset, just before $18^{\mathrm{h}}$, are times at which the greatest changes in ionospheric absorption occur, an effect which is characteristic of all stations, in some degree. At Delhi during the same month, however, it will be seen that the diurnal variation was negligible, being approximately three units as compared with approximately 30 units at Accra.

That there was an even greater drop in noise at Accra than these polygons indicate is at least suggested by the flattening of the monthly range of all frequencies during the middle of the day. The lesser serration of the arrays toward midday may be explained by assuming that the observations were limited by set noise which should exhibit less scatter than natural noise. Decision on this matter must await the installation of improved equipment. The graph for Delhi, on the other hand, shows considerable serration throughout the 24 hours for all frequencies except $20 \mathrm{Mc}$. Therefore the Delhi observations on those frequencles may be regarded as largely free from limitations of set noise. Contrasted with the Accra data the Delhi data exhibit little diurnal variation.

Figure 4 shows the diurnal curves for June and December, at Balboa. There, also, lack of serration in the graph for December 1945 suggests that the observations for that month were subject to set noise limitations, though the graph for June exhibits sufficient serration on the first three frequencies to indicate that the 90 per cent value was consistently enough above set noise to be significant throughout the day.

The curves superposed on the 2.5 and $5 \mathrm{Mc}$ graphs are the predictions of the United States Army Signal Corps given in their report RPU-140, a revision of the noise curves and charts in the Radio Propagation Handbook of the Interservice Radio Propagation Laboratory. The Signal Corps
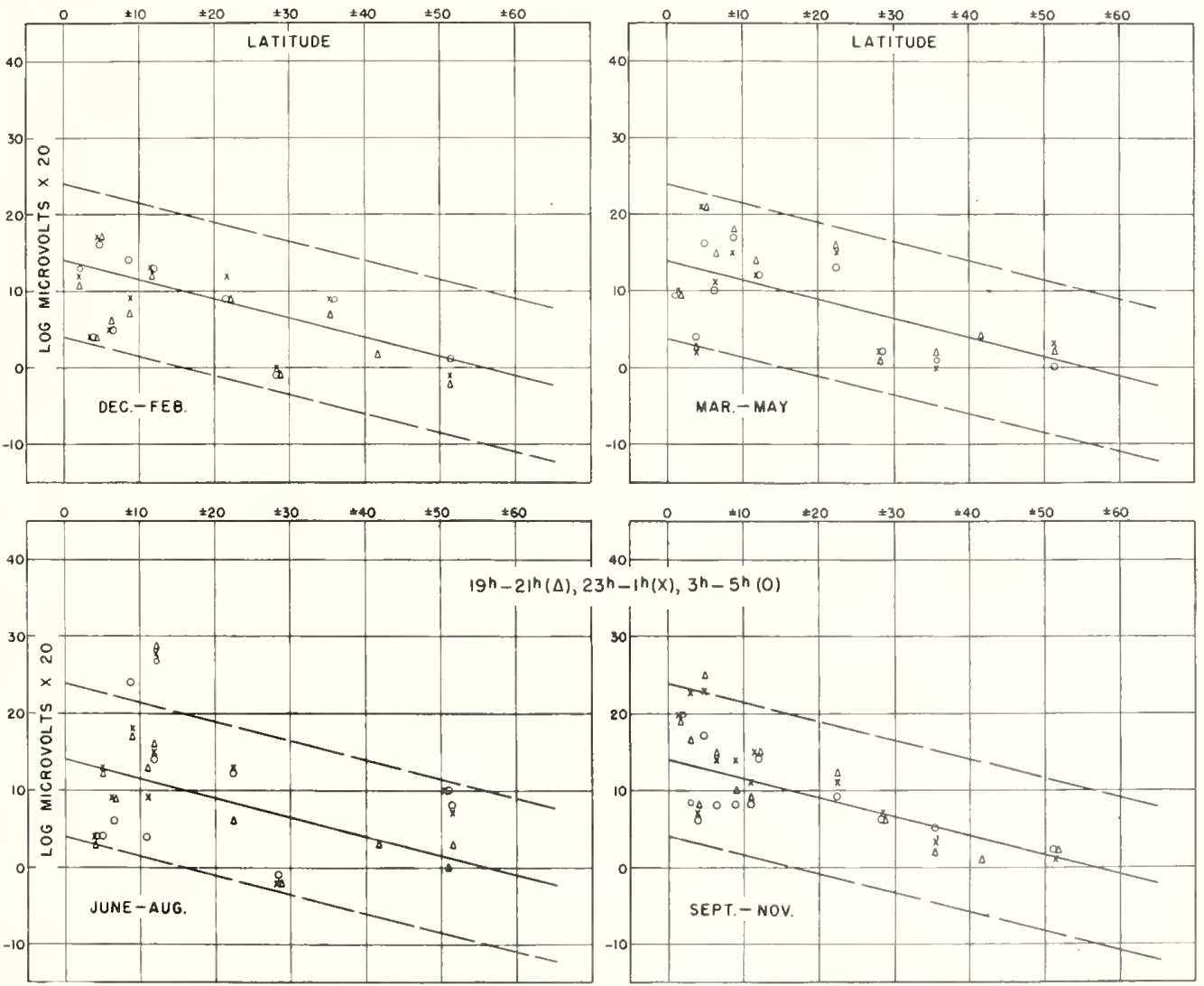

Fig. 5--Upper decile values of atmospheric radio noise at five Mc during night hours at various stations 
curves represent a value directly comparable with the observed 95 per cent intelligibility 90 per cent of the time. In both June and December at 2.5 and $5 \mathrm{Mc}$ the Balboa observations failed to go as low as the prediction. Again it is necessary to wait until more sensitive measuring equipment is in operation before deciding whether the daytime predictions are in error or the observed values are limited by set noise.

These two months of Balboa data indicate a well-defined seasonal variation with a drop of about 20 units from June to December. Though the supposition of thunderstorms origin of atmospheric radio noise would lead to expectation of a considerable seasonal variation in this direction, no consistent difference of this magnitude was exhibited by the nontropical stations, nor did all tropical stations show a comparable drop. A change of 7.5 units was the average for all stations from June 1946 to December 1946 on $2.5 \mathrm{Mc}$, but there was a rise of only 4.5 units from December 1945 to June 1946. It appears from this that the seaonal variation may not be as great as has been supposed. Allowing for consistently lowered levels from one year to the next, possibly an effect of solar activity on the propagation of atmospheric radio noise, the average variation which can be attributed to seasonal effects is only of the order of six units. On five Mc about the same seasonal variation in the averages is apparent.

The means of the upper decile values for all data from the various stations at $19 \mathrm{~h}$ to $21 \mathrm{~h}$, $03^{\mathrm{h}}$ to $05^{\mathrm{h}}$, and $23^{\mathrm{h}}$ to $01^{\mathrm{h}}$ are plotted by seasons in Figure 5 (for five Mc) and in Figure 6 (for 2.5 Mc). The solid lines are the least squares adjustment for latitude dependence, assuming linearity, obtained by lumping together the data for all seasons. Although better fits could have been obtained by separate adjustment for each season the improvement was not considered worth while in view of the greater simplicity of the representation used. As indicated in Figures 5 and 6 the latitudes of the stations have been handled without regard to sign, but where data for southern stations were used they have been plotted in the reverse season. The slopes of the lines indicate a decrease in noise with increasing latitude at rates of 0.24 and 0.36 unit per degree for 5 and 2.5 Mc respectivel
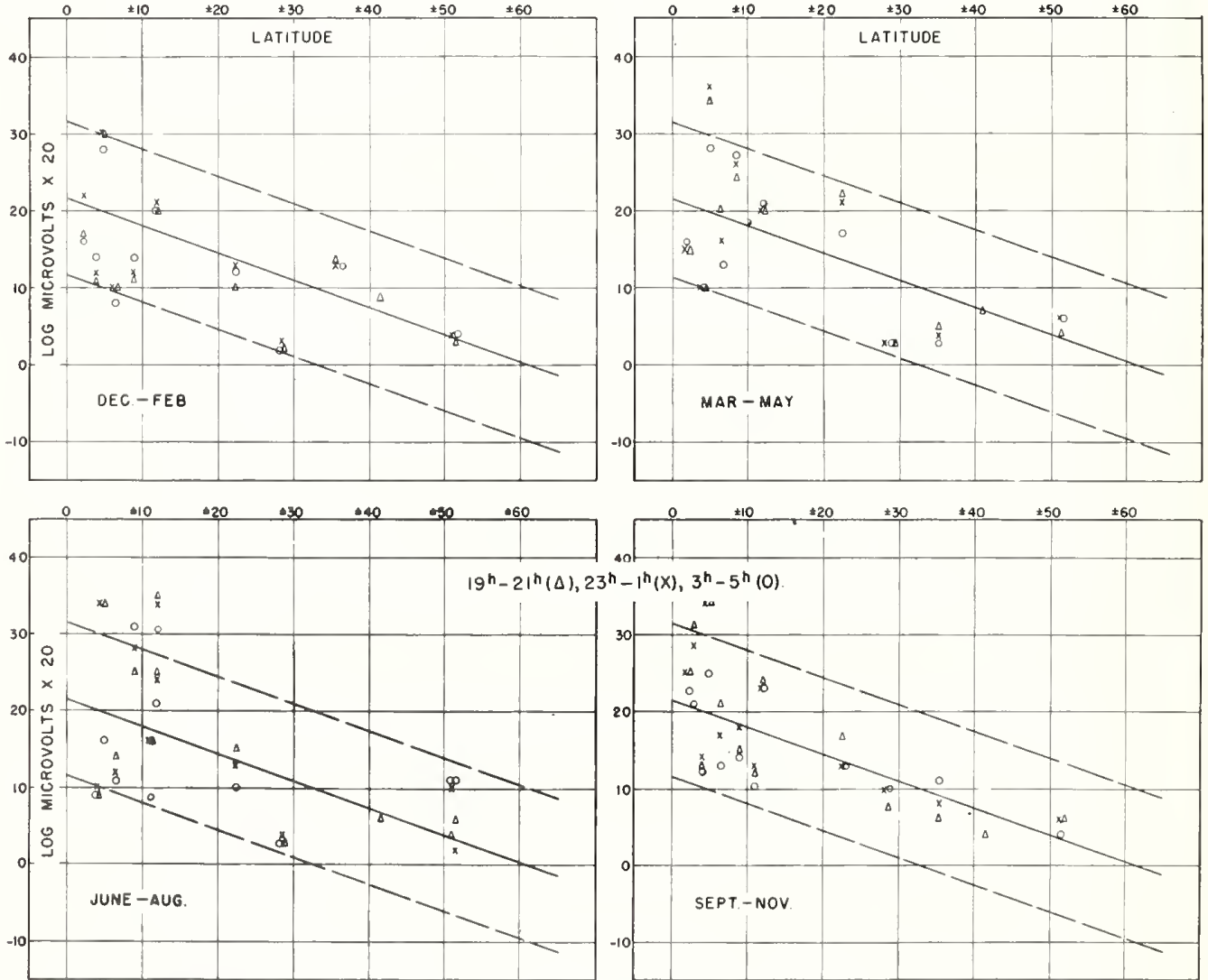

Flg. 6--Upper decile values of atmospheric radio noise at 2.5. Mc during night hours at various stations 
The dashed lines in Figures 5 and 6 represent a ten-unit departure from the latitude-variation line and include 93 per cent of all the points. The greatest scatter of points is in lower latitudes.

The least-square lines fitted by individual months to the data of Figure 6 for the $2.5 \mathrm{Mc}$ noise had various slopes and intercepts. The intercepts at latitude $25^{\circ} \mathrm{N}$ are plotted in Figure 7. In a certain sense these intercepts represent averages of the upper decile values of radio noise for the northern hemisphere. Distinct seasonal and long-term trends are exhibited by the plot, corresponding to a seasonal sine wave with an amplitude of about three units, with its maximum

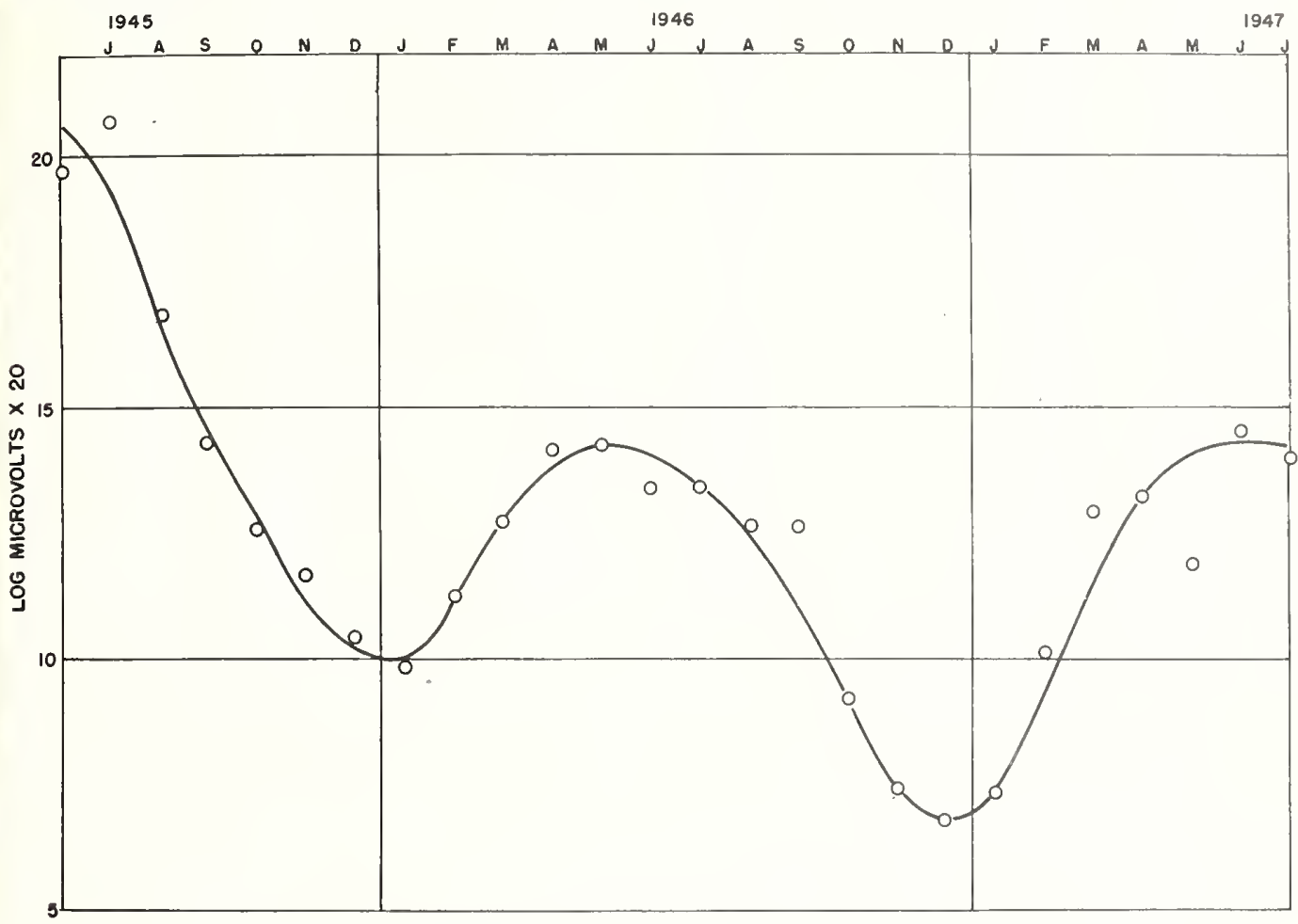

Fig. 7--Trend of monthly average of atmospheric noise at 2.5 Mc, Northern Hemisphere, June 1945 to July 1947

near the June solstice, superposed on a general decreasing trend at a rate of about four un ts per jear. More definite information on the cause of this effect will probably be obtained when the susspot maximum has been passed and the decreased solnr activity produces less absorption. It may be expected that the annual curve of noise level will gradually approach a true sine curve at the time of sunspot maximum and later will reverse its present trend. Similar effects are observed on five Mc, but they are less pronounced.

The average values of atmospheric radio noise and the values given by the least square lines of Figures 5 and 6 may be regarded as predictions, and as such may be compared with predictions made in other ways. Such comparisons of predicted and observed values on the $2.5 \mathrm{Mc}$ and five Mc frequencies are shown in Figure 8. The first pair of polygons shows the percentage of observations for the night hours, averaged as before by three-hour groupings, which fall within plus or minus 10, 20, and 30 units of the predictions of the Signal Corps report, RPU-140, calculated for each station. The second pair of polygons represents the same type of comparison assuming that the noise level the world over is 15 units on $2.5 \mathrm{Mc}$ and 10 on five Mc. These "predictions" are about as good as the Signal Corps predictions. The third pair of polygons represents the same kind of comparison, the "prediction" being given by the latitude variation lines in Figures 5 and 6. These results are slightly better than the Signal Corps predictions for $2.5 \mathrm{Mc}$ and five Mc. 


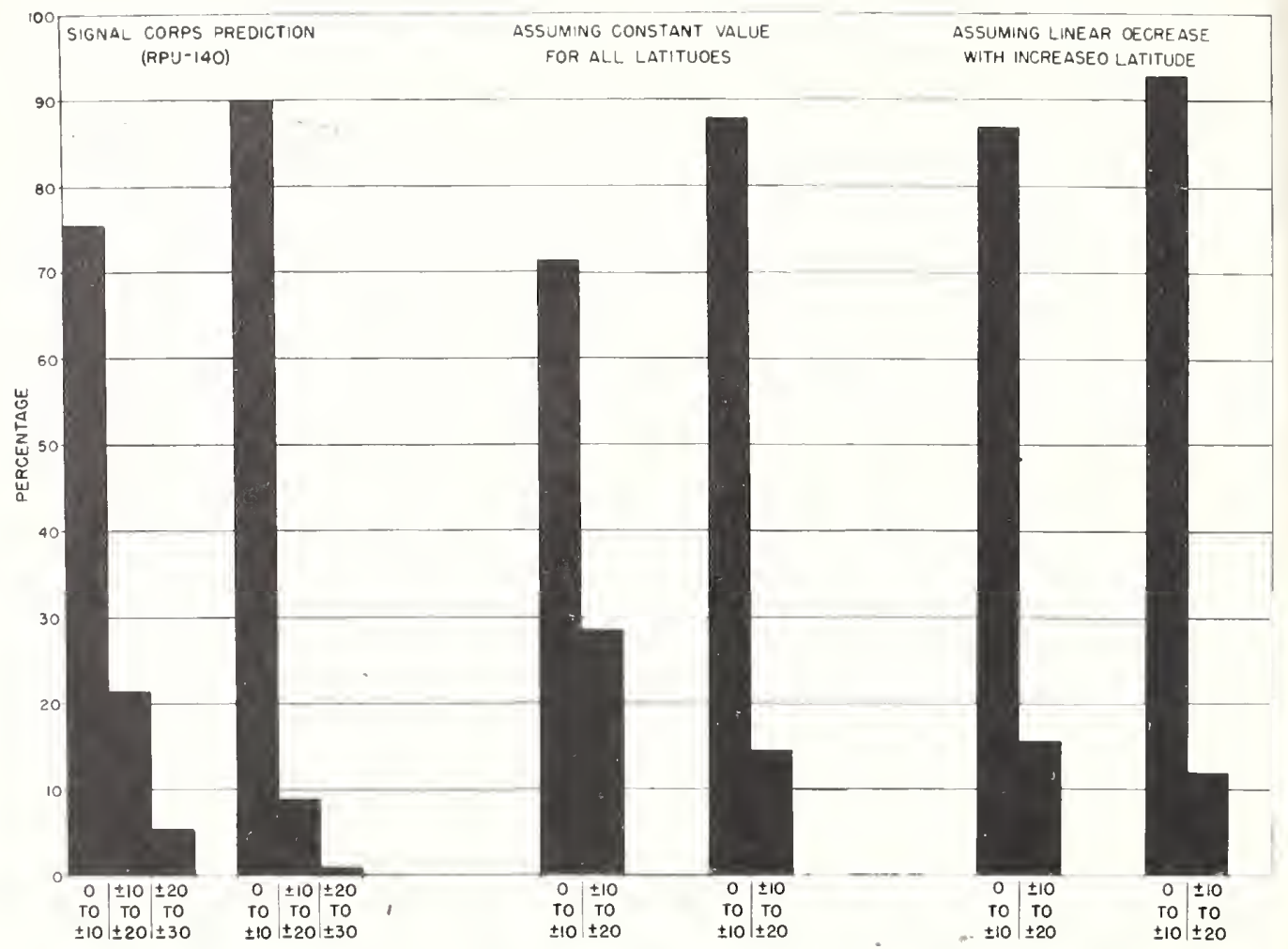

Fig. 8--Percentage of deviations of observed atmospheric radio noise from predictions

Since the parameters used in the first pair of polygons in the Figure were determined from the data themselves, somewhat better results were obtained than would be expected if the predictions were made for regions for which no data were available. It is of some interest to remark in this connection that Southern Hemisphere data, not available when the original adjustments were made, fell close to the values "predicted" from data taken in the Northern Hemisphere. The results do show that the observed world-wide distribution of atmospheric radio noise on 2.5 and five $\mathrm{Mc}$ at the hours and stations in question is about as well represented by assuming a simple linear latitude variation as by the much more complicated charts given by the Signal Corps in RPU-140. Improvement would have been obtained had the effects revealed in Figure 7 been taken into account; however, inclusion of them would have involved the use of a considerably larger number of parameters.

Central Radio Propagation Laboratory,

National Bureau of Standards, Washington, D. C.

(Manuscript recelved October 29, 1947; presented at the Joint Meeting of the URSI and IRE, Washington, D. C., May 6, 1947; open for formal discussion until May 1, 1948.) 

